\title{
Assessment of Passenger's Ride Comfort of a Tricycle
}

\author{
Ján Dižo ${ }^{1 *}$, Miroslav Blatnický ${ }^{1}$ and Rafał Melnik ${ }^{2}$ \\ ${ }^{1}$ University of Žilina, Faculty of Mechanical Engineering, Department of Transport and Handling \\ Machines, Univerzitná 8215/1, 01026 Žilina, Slovak Republic; Email: jan.dizo@fstroj.uniza.sk, \\ miroslav.blatnicky@fstroj.uniza.sk \\ ${ }^{2}$ Warsaw University of Technology, Faculty of Transport, Department of Design Fundamentals of \\ Transport Means, Koszykowa 75,00-662 Warszaw, Poland; Email: rme@wt.pw.edu.pl
}

\section{*Corresponding Author: Ján Dižo}

\begin{abstract}
Passenger ride comfort of road vehicles is one of the most important performances of vehicle. The research of automotive passenger ride comfort is getting more and more important. In this contribution, the multibody model of a three-wheeled vehicle and analyses of passenger ride comfort is presented. The vehicle model and dynamic analyses were performed using the Simpack software package. The dynamic analyses of the vehicle driving were carried out on the road model with different surface quality. Analyses were focused on assessment of the passenger ride comfort on a driver seat.
\end{abstract}

Keywords: Tricycle, passenger ride comfort, multibody system, computer simulations

\section{Introduction}

The introduction should put the focus of the manuscript into a broader context and should supply sufficient background information to allow the reader to understand and evaluate the results without referring to previous publications on the topic.

Smoothness is called the riding comfortableness. It is the main performance of the modern road vehicles with high efficiency. The target quality of the smoothness affects directly the performance display, the travel system's life, as well as human's working efficiency and health. So how to guarantee the road vehicle has good smoothness has already attracts designer's attention. In recent years, with development of the computer technology, the theory of random vibration, the testing method as well as the systems dynamics. Simulation of road vehicle ride comfort is to be more comprehensive and much closely approach to the fact.

The multibody model of a tricycle and dynamic analyses has been carried out in Simpack software package. In this software there is possible to create dynamic models of road vehicles, rail vehicles and many other kind of transport [1,2]. 


\section{Dynamic Model of a Tricycle}

The vehicle is a complex mechanical system which is made of wheels, suspension, vehicle body (or frame), drivetrain, transmission, steering system etc. When we discuss the passenger ride comfort, we mainly consider the vibration caused by the road surface roughness. The road input passes to the human body through the tire, suspension, the seat cushion, that is elastic damping part. These constitute the vehicle vertical vibration system interacted. In order to improve computing speed of the MBS model, we can simplify secondary influence factors to ride comfort under the condition in which the model is accurate.

From a mathematical point of view the multibody model of a three-wheeled vehicle is in the MBS software described and solved as a system of equations of motion, which we can write in standard second-order matrix form in the known formulation:

$$
M z^{\prime \prime}+B z^{\prime}+K z=F
$$

where $\boldsymbol{M}, \boldsymbol{B}$ and $\boldsymbol{K}$ are matrix of mass, damping and stiffness, respectively and $\boldsymbol{z}^{\prime \prime}, \boldsymbol{z}^{\prime}$ and $\boldsymbol{z}$ are vectors of accelerations, velocities and displacements, respectively.

Fig. 1 shows the virtual model of the analysed three-wheeled vehicle and the scheme of a simplified model of the analysed vehicle is shown in Fig. 2.
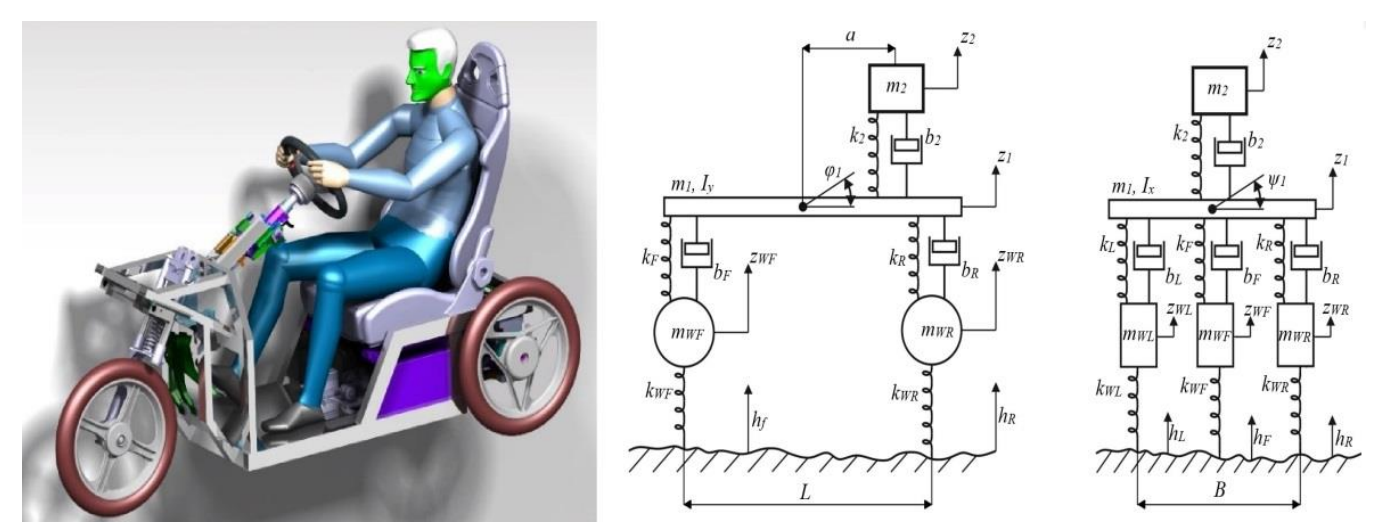

Fig. 1 Virtual model of a tricycle (left) and its simplified dynamic scheme (left). Source: authors

The object of this research is analysis of the passenger's ride comfort of a tricycle designed in as a prototype, which include two key factors: effective and ecological electric power-train and the newly designed steering system, which is possible to improve the overturning stability of the tricycle in curves. The whole structure of this vehicle is presented and particularly described in [3$5]$. 


\subsection{Suspension System of a Tricycle}

All suspension parts are modelled as linear elements. The multibody model of an analysed vehicle is a complex mechanical system which is made of wheels, suspension, vehicle body (or frame), drivetrain, transmission, steering system etc.

The front suspension of our considered three-wheeled vehicle in the base consists of a fork, a handlebar and one coil spring without damper. Damping is ensured by this spring. In the front part of the vehicle is installed steering system. It consists of several special designed parts, which ensured better overturning stability of this vehicle in comparison with standard steering system.

In the rear part of the vehicle the independent suspension of rear wheels is used. It consists of two swinging arm, a wheel hub and combination of coil spring and hydraulic damper on each side of the axle. To each rear wheel the axle drive shaft ensuring vehicle drive is linked. It means, the vehicle uses rear wheel drive.

\section{Entire Model of a Tricycle and a Road}

The Simpack software provides overall eight tire models for road vehicle from the simplest to the most complex. We have used the Pacejka Similarity model, which which represents complex multiforce modelling element.

Into the road model there is possible to include the excitation, which simulate more or less real irregularities of a road. The most often the user can define irregularities in the form of harmonic function or stochastic from power spectral density (PSD). In our research for a road model we have used stochastic excitation in the form of PSD. This functionality creates a pseudo stochastic road excitation signal in distance domain in the basis of PSD function.

This takes a PSD as input and creates on this basis a pseudo stochastic signal in distance domain, which can be directly applied to the road model. The modelling elements that provide the contact of the wheel to the road will convert the distance domain excitation into the according time domain excitation according to their current driving speed [6].

The PSD defines how the total power of the considered process is distributed in the whole frequency range. It means, by integration of the PSD for all excitation frequency we obtain the RMS value of the investigated random process. The PSD is the Fourier transformation of the autocorrelation process and it is given as:

$$
S_{h}(\omega)=\int_{-\infty}^{\infty} K_{h}(\tau) e^{-i \omega \tau} d \tau
$$


where $S_{h}(\omega)$ is the power spectral density (PSD) of road irregularities, $K_{h}(\tau)$ is the autocorrelation function of road irregularities, $\tau$ is time range and $\omega$ is the angular frequency of excitation [7].

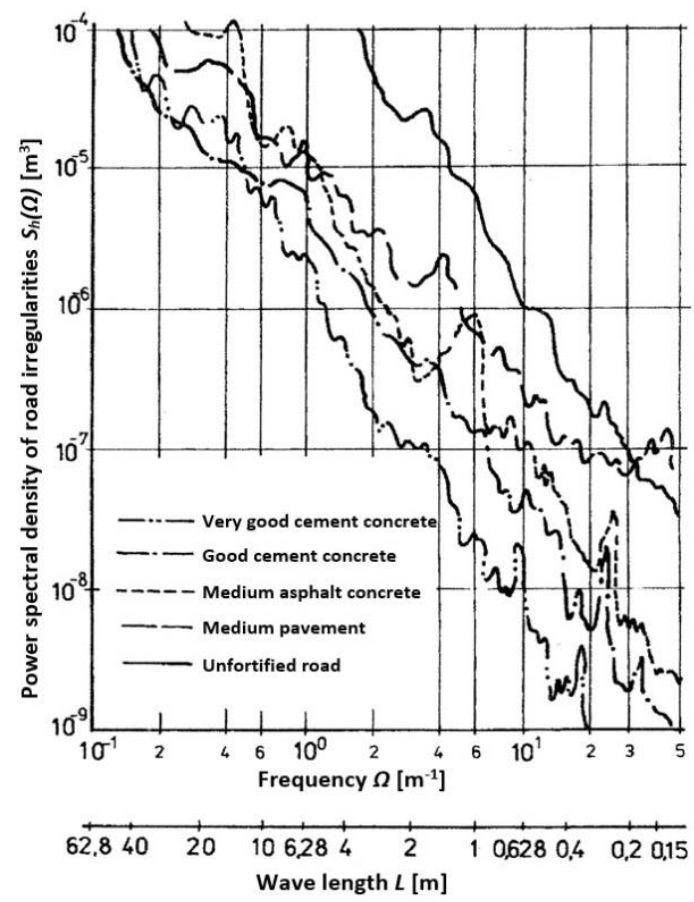

Fig. 2 Example of the PSD of various road qualities. Source: [7]

The PSD of road irregularities is obtained by experiments. Road irregularities are usually measured by a test vehicle with the special trailer and post-processed in order to have graphical and numerical outputs from the measurements. An example of experimental measurements outputs of various road surfaces qualities is shown in Fig. 2.

From the used software library we have chosen seven types of predefined PSD, which correspond to various qualities of road surfaces [6]. For purposes of simulation computations, these surfaces are marking in compliance with Table 1.

Table 1 Scale for evaluation of ride comfort for passenger. Source: [6]

\begin{tabular}{cc}
\hline Road quality & Marking \\
\hline Very good cement concrete & $\mathrm{A}$ \\
\hline Good cement concrete & $\mathrm{B}$ \\
\hline Good asphalt concrete & $\mathrm{C}$ \\
\hline Good macadam & $\mathrm{D}$ \\
\hline Medium asphalt concrete & $\mathrm{E}$ \\
\hline Medium pavement & $\mathrm{F}$ \\
\hline Bad pavement & $\mathrm{G}$ \\
\hline
\end{tabular}




\section{Simulation Computations and Results}

In our work we analysed the three-wheeled vehicle model, which was run on a straight road model. The road model includes irregularities modelled as power spectral density, as it was just described above.

In our research we have wanted to investigate dynamic effects of the driving such the vehicle on passenger's ride comfort of vehicle, which has run under various operational conditions. As the power-train of the analysed vehicle is designed for the maximal speed of $15 \mathrm{~km} / \mathrm{h}$ and the technical modification of the power-train of vehicle allows to increase the maximal speed up to $30 \mathrm{~km} / \mathrm{h}$ and considering the main use of it is considering in urban transport, we have chosen range of speeds from $10 \mathrm{~km} / \mathrm{h}$ to $30 \mathrm{~km} / \mathrm{h}$. Analyses were recorded at corresponding sampling rates depending on speeds. Moreover we have also evaluated various road conditions, i. e. road quality described in the previous chapter.

Recently, the analysed three-wheeled vehicle is designed for one passenger (it means for a driver). As the criteria of the passenger ride comfort assessment of the vehicle driving on the road under various operational conditions we have chosen values of accelerations measured on the driver seat, because evaluation of acceleration values in this location is common criterion entrenched in the standard.

We have recorded data for various speeds of driving and road quality in time domain. It means we have relatively large number of simulations of the vehicle driving. Therefore, for the presentation of results we have chosen outputs in such a form, which visual illustrates monitored quantities in partial range. Furthermore, we have also statistically processed outputs of accelerations [8-10] according to the ISO 2631:1997.

The weighting acceleration root mean square value in $x, y$ and $z$ directions, can be obtained by integral equation of the auto power spectral density function which this longitudinal vibration acceleration function is:

$$
a_{W_{i}}=\sqrt{\int_{80}^{0.5} W_{i}^{2}(f) \cdot G_{a_{i}}(f) \cdot d f}
$$

where $a_{w_{i}}$ is weighting acceleration root mean square value in $x, y$ and $z$ directions $(i=x, y, z)$, $W_{i}^{2}(f)$ is frequency weighting function in $x, y$ and $z$ directions $(i=x, y, z)$ and $G_{a_{i}}(f)$ is weighting acceleration power spectral density function in $x, y$ and $z$ direction $(i=x, y, z)$.

Finally, the total weighted acceleration is determined following: 


$$
a_{r m s, w}=\sqrt{a_{x, m m s, w}^{2}+a_{y, r m s, w}^{2}+a_{z, r m s, w}^{2}},
$$

The standard above-mentioned ISO specification defines a scale for evaluation of ride comfort for passenger. It is listed in Table 2.

Table 2 Scale for evaluation of ride comfort for passenger. Source: [8,9]

\begin{tabular}{cc}
\hline Total $a_{r m s, w}\left[\mathbf{m} / \mathbf{s}^{2}\right]$ & Perception \\
\hline$<0.315$ & not uncomfortable \\
\hline 0.315 to 0.63 & a little uncomfortable \\
\hline 0.5 & fairly uncomfortable \\
\hline $0.8-1.6$ & uncomfortable \\
\hline $1.25-2.5$ & very uncomfortable \\
\hline$>2$ & extremely uncomfortable \\
\hline
\end{tabular}

Let's compare values of calculated weighted accelerations $a_{r m s, w}$ from Table 3 with scale for evaluation of passenger's ride comfort listed introduced in Table 2. We can identify, that the driving of the tricycle on the road of „A“" surface quality at the speed of $10 \mathrm{~km} / \mathrm{h}$ and $15 \mathrm{~km} / \mathrm{h}$ is tolerably comfortable. It corresponds with the designed maximal driving speed of the analysed vehicle. But, if we investigate comfort for greater speed, we can see, that driving on a road of good quality is much less comfortable with increasing driving speed. And also, driving of the vehicle on the worse road quality, mainly on the "F" and "G" is very uncomfortable, even dangerous for human health. It shows important finding.

Table 3 Calculated total weighted acceleration $a_{r m s, w}$ for different various driving conditions.

Source: authors

\begin{tabular}{|c|c|c|c|c|c|}
\hline Speed & $10 \mathrm{~km} / \mathrm{h}$ & $15 \mathrm{~km} / \mathrm{h}$ & $20 \mathrm{~km} / \mathrm{h}$ & $25 \mathrm{~km} / \mathrm{h}$ & $30 \mathrm{~km} / \mathrm{h}$ \\
\hline Road quality & $a_{r m s, w}\left[\mathbf{m} / \mathbf{s}^{2}\right]$ & $a_{r m s, w}\left[\mathbf{m} / \mathbf{s}^{2}\right]$ & $a_{r m s, w}\left[\mathbf{m} / \mathbf{s}^{2}\right]$ & $a_{r m s, w}\left[\mathbf{m} / \mathbf{s}^{2}\right]$ & $a_{r m s, w}\left[\mathbf{m} / \mathbf{s}^{2}\right]$ \\
\hline A & 0.649 & 0.844 & 1.583 & 3.003 & 7.680 \\
\hline B & 1.058 & 0.935 & 1.622 & 3.023 & 7.655 \\
\hline $\mathrm{C}$ & 0.812 & 0.907 & 1.659 & 3.113 & 6.821 \\
\hline D & 0.863 & 1.022 & 1.885 & 3.473 & 7.286 \\
\hline$E$ & 2.223 & 1.515 & 2.566 & 4.653 & 6.033 \\
\hline $\mathrm{F}$ & 3.131 & 2.063 & 2.863 & 4.605 & 5.674 \\
\hline $\mathrm{G}$ & 3.786 & 3.134 & 3.056 & 4.588 & 7.292 \\
\hline
\end{tabular}

Taking account of the original designation of electric tricycle, which our new design goes out from, we can state, that construction and parameters of current suspension system of the analysed 
tricycle is acceptable for lower driving speed (up to $15 \mathrm{~km} / \mathrm{h}$ ) and for relatively good road quality, i. e. good asphalt concrete or good macadam, for which the compared values of calculated total weighted acceleration is still acceptable.

Our intention with the designed electric tricycle is making the most of advantages of our steering system. Therefore we are working on such a power-train, which allow increasing the maximal driving speed of the tricycle. But, from the obtained results it will not possible without improving the suspension system [11-13] in such a way, that the passenger's ride comfort will be on the acceptable level throughout allowed range of speed.

\section{Conclusion}

Through the survey and evaluation method in international ISO 2631 standard, considering the influence of the different road qualities as well as driving speeds, we may reflect comprehensive and accurately how comfortable the passenger feels riding our designed tricycle. After above presented results from simulation analyses analysis we can identify, when the vehicle is more and when the vehicle is less comfortable. Hereby we have found out, that current construction of the tricycle does not meet safety and comfort requirements and a modification of the design is necessary.

\section{Acknowledgments}

This section should describe sources of funding that have supported the work. This work was supported by the Cultural and Educational Grant Agency of the Ministry of Education of the Slovak Republic in project No. KEGA 077ŽU-4/2017: Modernization of the Vehicles and engines study program.

\section{References}

[1] Hauser, V., Nozhenko, O., Kravchenko, K., Loulova, M., Gerlici, J \& Lack, T. (2017). Proposal of a steering mechanism for tram bogie with three axle boxes. Procedia Engineering 192, 289-294. DOI: 10.1016/j.proeng.2017.06.050.

[2] Hauser, V., Nozhenko, O., Kravchenko, K., Loulova, M., Gerlici, J. \& Lack, T. (2017). Impact of three boxes bogie to the tram behavior when passing curved track. Procedia Engineering 192, 295-300. DOI: 10.1016/j.proeng.2017.06.051.

[3] Kurčík, P., Harušinec, J., Strážovec, P \& Pavlík, A. (2018). Strength check of the E3-kolka frame (In Slovak). In Experimental and Computational methods in engineering 2018: 5th conference for young scientific workers, 6-8 June 2018 (pp. 62-69). Ústí nad Labem, Czech Republic: J. E. Purkyně University. 
[4] Pavlík, A., Dižo, J., Kurčík, P \& Strážovec, P. (2018). Improvement driving characteristics of the electric tricycle. In Experimental and Computational methods in engineering 2018: 5th conference for young scientific workers, 6-8 June 2018 (pp. 70-77). Ústí nad Labem, Czech Republic: J. E. Purkyně University.

[5] Strážovec, P., Kurčík, P., Pavlík, A. \& Blatnický, M. (2018). E3-kolka with a steering mechanism for improvement of the stability in curving. In Experimental and Computational methods in engineering 2018: 5th conference for young scientific workers, 6-8 June 2018 (pp. 107-114). Ústí nad Labem, Czech Republic: J. E. Purkyně University.

[6] Kardas-Cinal, E. (2018). Methods of vibrational comfort evaluation in means of transport. In Transport Means 2018: $22^{\text {nd }}$ International Scientific Conference, Part II, 12-14 October 2018 (pp. 1008-1013). Trakai, Lithuania: Kaunas University of Technology.

[7] Kardas-Cinal, E. (2010). Ride comfort for various passenger positions in a railway vehicle Simulation study. Archive of Transport 22 (2), 189-199. DOI: 10.2478/v10174-010-0012-3.

[8] Vlk, F. (2003). Dynamics of engine vehicles (In Czech) (2 $2^{\text {nd }}$ ed.). Brno, Czech Republic: Prof. Ing. František Vlk, DrSc., nakladatelství a vydavatelství.

[9] ISO 2631:1997. Mechanical vibration and shock. Evaluation of human exposure to wholebody vibration. Part I: general Requirements. International Organization for Standardization.

[10] Simpack Documentation (2018). Simpack User's Manual.

[11] Lugner, P. \& Edelmann, J. (2018). An approach for minimizing the number of objective functions in the optimization of vehicle suspension systems. Journal of Sound and Vibration 435, 149-169. DOI: 10.1016/j.jsv.2018.08.009WoS.

[12] Ning, D., Sun, S, Du, H., Li, W. \& Li, W. (2018). Control of a multiple-DOF vehicle seat suspension with roll and vertical vibration. Journal of Sound and Vibration 435, 170-191. DOI: $10.1016 /$ j.jsv.2018.08.005.

[13] Yang, Z., Yong, C., Li, Z. \& Yin, K. (2018). Simulation analysis and optimization of ride quality of in-wheel motor electric vehicle. Advanced in Mechanical Engineering 10(5), 10 pages. DOI: $10.1177 / 1687814018776543$. 